\title{
German Vegetation Reference Database (GVRD)
}

\author{
Ute Jandt \& Helge Bruelheide
}

\begin{abstract}
The German Vegetation Reference Database (GVRD; GIVD ID EU-DE-014) was established in 2008 as a reference vegetation database for vegetation records of the German Biodiversity Exploratories (http://www.biodiversity-exploratories.de/). Initially, the database had a focus on forest and grassland vegetation but is currently undergoing a continuous broadening of scope. Relevés were digitized and compiled from published and unpublished sources and from various thematic, regional or local databases with the ambition to cover the whole territory of Germany. The database is hosted at the Martin-Luther University Halle-Wittenberg at the Institute of Biology in the Department of Geobotany and is one of the database platforms of the German Centre of Integrative Biodiversity Research (iDiv, http://www.idiv-biodiversity.de). The functionality of the database is increasingly enhanced, currently comprising links to Germany's trait databases and GIS. At present, the database is used to test current theories in community assembly rules (http://www.biodiversity-exploratories.de/1/projects/weitere-projekte/botany-related-studies/berich/). Further current analyses are the temporal and geographical distribution of species and of vegetation types and the assessment of biodiversity.
\end{abstract}

Keywords: Germany; National Reference Database; vegetation database.

GIVD Database ID: EU-DE-014

Last update: $2012-07-11$

\section{German Vegetation Reference Database (GVRD)}

Scope: German Vegetation Reference Database (GVRD) of the German Biodiversity Exploratories and others. Main focus on forest and grassland vegetation. Relevés digitized and compiled from published and unpublished sources and from various thematic, regional or local databases.

Status: ongoing capture

Period: $1895-2011$

Database manager(s): Ute Jandt (ute.jandt@botanik.uni-halle.de); Helge Bruelheide (helge.bruelheide@botanik.uni-halle.de)

Owner: [NA]

Web address: http://www.biologie.uni-halle.de/bot/vegetation_db//index.php?Lang=E

Availability: according to a specific agreement

Online upload: no Online search: yes

Database format(s): TURBOVEG, MS Access, MySQL

Export format(s): TURBOVEG, MS Access, SQL, Excel, CSV file, plain

Publication: [NA]

Plot type(s): normal plots text file

Non-overlapping plots: 111,928

Plot-size range: $1-5000 \mathrm{~m}^{2}$

Total plot observations: 111,928

Estimate of existing plots: [NA]

Completeness: [NA]

Countries: DE: 99.9\%

Forest: [NA] — Non-forest: [NA]

Guilds: all vascular plants: $100 \%$; bryophytes (terricolous or aquatic): $49 \%$; lichens (terricolous or aquatic): $7 \%$; algae (terricolous or aquatic): $1 \%$

Environmental data: altitude: $61 \%$; slope aspect: $45 \%$; slope inclination: $48 \%$; soil depth: $5 \%$; soil pH: $8 \%$; other soil attributes: $9 \%$; land use categories: $1 \%$

Performance measure(s): cover: $100 \%$

Geographic localisation: small grid (not coarser than $10 \mathrm{~km}$ ): $87 \%$; political units or only on a coarser scale (>10 km): $90 \%$

Sampling periods: < 1919: 1.0\%; 1920-1929: 0.1\%; 1930-1939: 1.6\%; 1940-1949: 1.1\%; 1950-1959: 7.1\%; 1960-1969: 11.4\%; 1970-1979:

11.7\%; 1980-1989: 17.4\%; 1990-1999: 30.9\%; 2000-2009: 7.3\%; 2010-2019: 0.2\%; unknown: $10.2 \%$

Information as of 2012-07-12; further details and future updates available from http://www.givd.info/ID/EU-DE-014

Ute Jandt* (ute.jandt@botanik.uni-halle.de), Helge Bruelheide (helge.bruelheide@ botanik.uni-halle.de)

Institute for Biology, Geobotany and Botanical Garden, Martin-Luther-University Halle-Wittenberg,Am Kirchtor 1, 06108 Halle

(Saale), GERMANY

*Corresponding author 